\title{
Erratum to: Adenoviral transfer in the liver: a novel in vivo simulation model in the rat
}

\section{E. Matevossian, V. Assfalg, A. Novotny, D. Reim, Ch. Schmaderer, H. D. Kern, J. Nährig,} D. Doll, N. Hüser

Erratum to: Eur Surg

DOI $10.1007 / \mathrm{s} 10353-010-0554-\mathrm{x}$

Conflict of interest

The authors declare that there is no conflict of interest.

The version of the original article can be found under DOI: $10.1007 /$ s10353-010-0554-x

Correspondence: Edouard Matevossian, M.D., Department of Surgery, Klinikum rechts der Isar, Technical University Munich, Ismaninger Straße 22, 81675 Munich, Germany.

Fax: +49-89-4140-4805

E-mail: matevossian@chir.med.tu-muenchen.de

Medieninhaber und Herausgeber: Springer-Verlag GmbH, Sachsenplatz 4-6, 1201 Wien, Österreich. - Datenkonvertierung und Umbruch: Thomson Press (India) Ltd., Chennai; Druck: FRIEDRICH VDV, Vereinigte Druckereien- und Verlags-GmbH \& Co KG, 4020 Linz, Österreich. - Verlagsort: Wien. - Herstellungsort: Linz. 